\title{
Lagged Coherence of Photon Emissions and Spectral Power Densities between the Cerebral Hemispheres of Human Subjects during Rest Conditions: Phase Shift and Quantum Possibilities
}

\author{
J. N. Costa ${ }^{1,2}$, B. T. Dotta ${ }^{1,3}$, M. A. Persinger ${ }^{1,2,3,4^{*}}$ \\ ${ }^{1}$ Behavioural Neuroscience Program, Laurentian University, Sudbury, Canada \\ ${ }^{2}$ Biology Department, Laurentian University, Sudbury, Canada \\ ${ }^{3}$ Psychology Department, Laurentian University, Sudbury, Canada \\ ${ }^{4}$ Biomolecular Sciences Program, Laurentian University, Sudbury, Canada \\ Email:Jx_Costa@laurentian.ca,bx_dotta@laurentian.ca, *mpersinger@laurentian.ca
}

Received 14 February 2016; accepted 3 May 2016; published 6 May 2016

Copyright @ 2016 by authors and Scientific Research Publishing Inc.

This work is licensed under the Creative Commons Attribution International License (CC BY). http://creativecommons.org/licenses/by/4.0/

(c) (i) Open Access

\begin{abstract}
Photon counts about $15 \mathrm{~cm}$ from the left and right sides of the head while subjects sat quietly during baseline conditions within a hyper-dark chamber were measured by photomultiplier units. Lag/lead analyses for photon emissions between the two hemispheres indicated a weak but statistically significant correlation between the amplitude fluctuations that were separated by about 800 to $900 \mathrm{~ms}$. Analyses of the spectral power densities of photon amplitude variations from the left and right hemispheres revealed peak values between 2 and $3 \mathrm{~Hz}$ which were equivalent to a difference of about $900 \mathrm{~ms}$. The radiant flux densities were estimated to be in the order of 10-12 $\mathbf{W} \cdot \mathbf{m}^{-2}$ and to include the equivalence of about $10^{7}$ neurons. Our calculations, which accounted for the small magnitude of the strength of the interhemispheric coefficients, suggest that the coherence could be strongly correlated with processes associated with the unmyelinated axons with diameters between 400 to $800 \mathrm{~nm}$, the visible wavelengths, within the corpus callosum. When the ratio of the phase shift was applied to the Aharanov-Bohm equation, the time required for a photon-related electron to be within a cerebral magnetic field was the same duration as a single orbit of an electron and a photon's traversal latency across a neuronal plasma membrane. We suggest that the peak photon decoherence between the two cerebral hemispheres may reveal a neuronal-quanta substrate to the conditions associated with consciousness.
\end{abstract}

${ }^{*}$ Corresponding author.

How to cite this paper: Costa, J.N., Dotta, B.T. and Persinger, M.A. (2016) Lagged Coherence of Photon Emissions and Spectral Power Densities between the Cerebral Hemispheres of Human Subjects during Rest Conditions: Phase Shift and Quantum Possibilities. World Journal of Neuroscience, 6, 119-125. http://dx.doi.org/10.4236/wjns.2016.62015 


\section{Keywords}

\section{Biophotons, Interhemispheric Correlations, Imagination, Human Brain, Quantitative EEG (QEEG) Visualization, Corpus Callosum, Aharanov-Bohm Effect}

\section{Introduction}

The measurements of ultraweak light emissions from the right hemisphere of a human subject sitting in hyperdark settings $\left(10^{-12} \mathrm{~W} \cdot \mathrm{m}^{-2}\right)$ during periods of imagining white light compared to more mundane cognitions [1] indicated that complex neuronal processes associated with consciousness could be coupled to biophotons [1]-[3]. The strong correlation between quantitative electroencephalographic activity over the left prefrontal region and the flux density of photons emitted from the right hemisphere during these conditions suggests that intention and other forms of self-monitoring behaviours could potentially modify photon emissions. Biophotons are emitted from bacteria [4], cells [5], and hippocampal slices [6]. These electromagnetic emissions within the visible wavelength may be forms of intercellular communication rather than simply metabolic artifacts [7].

The seminal research of Popp [8] [9] and the Van Wijks [10] [11] has shown that photon emissions are measureable from several loci of the body, including the hands and head. The magnitudes, often measured in the order of $10^{7}$ photons per meter-squared per second, are effectively the same flux density we have measured from cells and the heads of human volunteers. Although photon emissions from the head might be attributed to sources within the hair or scalp rather than the cerebrum this explanation would not accommodate the reliable, reversible increase and decrease in photon counts or radiant flux densities coupled to imagining white light compared to mundane thoughts. These intervals are determined by the instruction of the experimenter and hence are less likely to be spurious associations.

To reduce the possibility of artifact and to further pursue the relationship between the characteristics of photon emissions from the left and right hemispheres we designed an experiment where photon emissions from both sides of the head were measured simultaneously. The primary goal was to discern if there is temporal coherence between the two hemispheres. In previous studies we [12] had found that electroencephalographic coherence between the left and right temporal lobe structures was affected by global geomagnetic activity, suggesting that relatively small amounts of energy could be involved with this enhanced intercalation. Here we present evidence for the first time that the amplitude variations in photon emissions from the left and right hemispheres are coherent but phase-lagged during resting (baseline) conditions.

\section{Materials and Methods}

To test this relationship seven subjects ( 3 men, 4 women) between 20 and 26 years of age volunteered as subjects after the protocol was approved by the university's research ethics committee. This small sample was considered sufficient because each person was employed as his or her "own" control and we have shown biophoton emissions from cerebral activity to be reversibly controlled by external instruction [1]. Photon emissions were measured from the left and from the right hemisphere simultaneously. The purpose of the experiment was to discern the temporal correlation between amplitudes of photon emissions, as measured by photomultiplier units, from the left and the right hemisphere. Vivid cognition or the act of imagining white light while sitting blind folded in a hyper-dark chamber $\left(10^{-12} \mathrm{~W} \cdot \mathrm{m}^{-2}\right)$ has been shown to significantly increase photon emissions. Consequently we decided to only compare bilateral photon emissions during baseline conditions when no active or at least instructed cognition was in progress. In other words is there an intrinsic decoherence or coherence in spectral density profiles between the two cerebral hemispheres of human beings when they simply sit in the dark and engage in cognition commensurate with the default network?

The procedures were very similar to those reported by Dotta et al. [1]. Each participant sat within a comfortable arm chair within a hyper-dark $\left(10^{-12} \mathrm{~W} \cdot \mathrm{m}^{-2}\right)$ acoustic chamber $\left(13 \mathrm{~m}^{3}\right)$. Digital photomultiplier devices were placed on the left and right side of the subject's head approximately $15 \mathrm{~cm}$ from the temporal lobes. The information from each device was captured by its own software and IBM laptop computer that was housed outside the chamber in a room that was also dark. The photons were sampled at 10 times per second for 1 min. To control for idiosyncratic features of a given photomultiplier unit, the location of the units (right or left side of the 
head) were counterbalanced for different subjects.

The numbers of photons per $100 \mathrm{~ms}$ ( $10 \mathrm{~Hz}$ sampling) for the middle $60 \mathrm{~s}$ (600 cases) of the baseline from the left and right side of the head for each subject were extracted and analyzed by SPSS (16) software. For each subject the photon numbers from the left and from the right side of the head were correlated. In order to discern potential temporal displacement between the two hemispheres the photon counts from the left and right hemisphere were lagged by 20 increments (100 to $2000 \mathrm{~ms}$ ) and then correlated with the reference unit for the other hemisphere.

\section{Results}

The results are shown in Figure 1. Although the strengths of the correlation coefficients were small and explained less than $1 \%$ of the variance the strongest significant relationship was the increase in photon radiant power from the left hemispheres about $900 \mathrm{~ms}$ before increased emissions from the right hemispheres. In fact the correlations with most of the other lags for the right hemisphere with respect to the left hemisphere and vice versa were significantly weaker as indicated by the overlap with the standard errors of the means.

It may be relevant that the numbers of corpus callosum fibers, each emerging from a single neuron, within the human brain constitute $1 \%$ or less of the total numbers of cortical neurons which average about 23 billion [13]. If the source of the approximately $1 \mathrm{~s}$ lag between the left and right hemispheric photon emissions was related to interhemispheric processing superimposed upon background biophoton emissions than one would expect this magnitude of strength of association. The upper limit for the estimated recurrent interhemispheric times for unmyelinated ( 0.1 to $1 \mu \mathrm{m}$ ) axons as described by Aboitiz et al. [13] would be about $900 \mathrm{~ms}$ which is well within

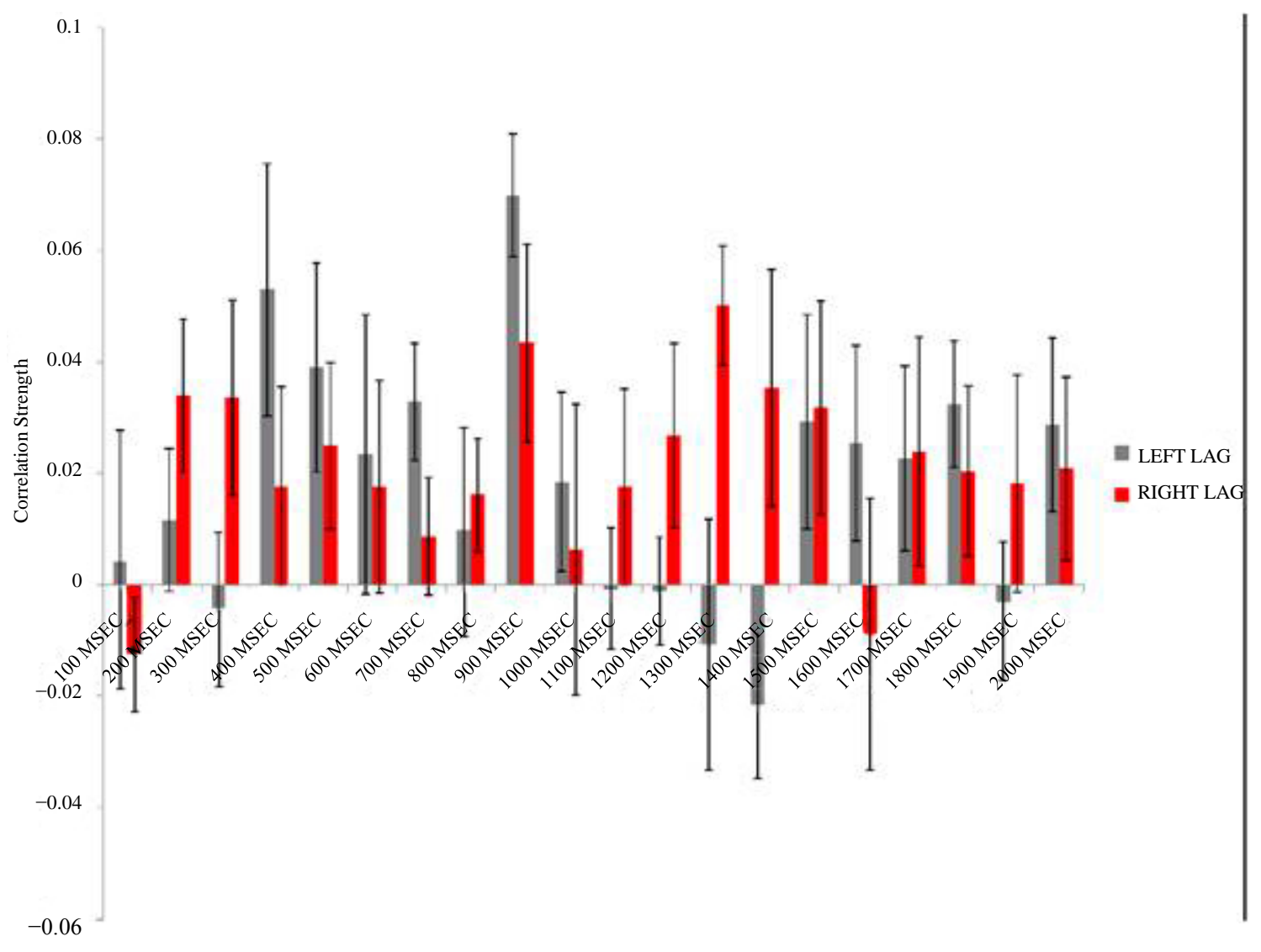

Figure 1. Correlation coefficients between the amplitude variations in photon emissions from the left and right hemisphere while subjects sat quietly in a hyper-dark room. The gray bars indicate the left hemisphere is temporally lagged behind the right hemisphere photon values. The red bars indicate the right hemisphere was lagged behind the left hemisphere. The horizontal axis indicates increments (in ms) of the lags. Vertical bars represent standard errors of the mean. 
the range of what we observed.

We reasoned that if this temporal displacement were valid then spectral analyses of the amplitude fluctuations in photon emissions should verify the effects. Spectral analyses (SPSS 16, Spectra) were completed for each of the seven subjects. The spectral power for the fluctuations in photon spectral power density from the right and left hemisphere for a sample participant is shown in Figure 2. All subjects displayed similar patterns. Spectral analyses revealed a peak in power density at $2.7 \mathrm{~Hz}$ for the left hemisphere and $1.85 \mathrm{~Hz}$ for the right hemisphere. There were no statistically significant correlations between the power densities.

In order to allow direct comparison the photon numbers from each hemisphere were z-scored before the spectral densities were calculated. The spectral power densities were compared. Lag correlations by the increments of $\Delta \mathrm{f}$ (in this case $\Delta \mathrm{f}=0.016 \mathrm{~Hz}$, the Nyquist Limit for the spectral power densities) were then computed for the spectral densities as a function of frequency bin for the data from each hemisphere. When they were lagged by $0.98 \mathrm{~Hz}$ to $1.15 \mathrm{~Hz}$ statistically significant negative correlations were clearly evident with a peak value of $\mathrm{r}=$ -0.23 . The results of the lag analyses are shown in Figure 3. This indicated that the average "out of phase" time

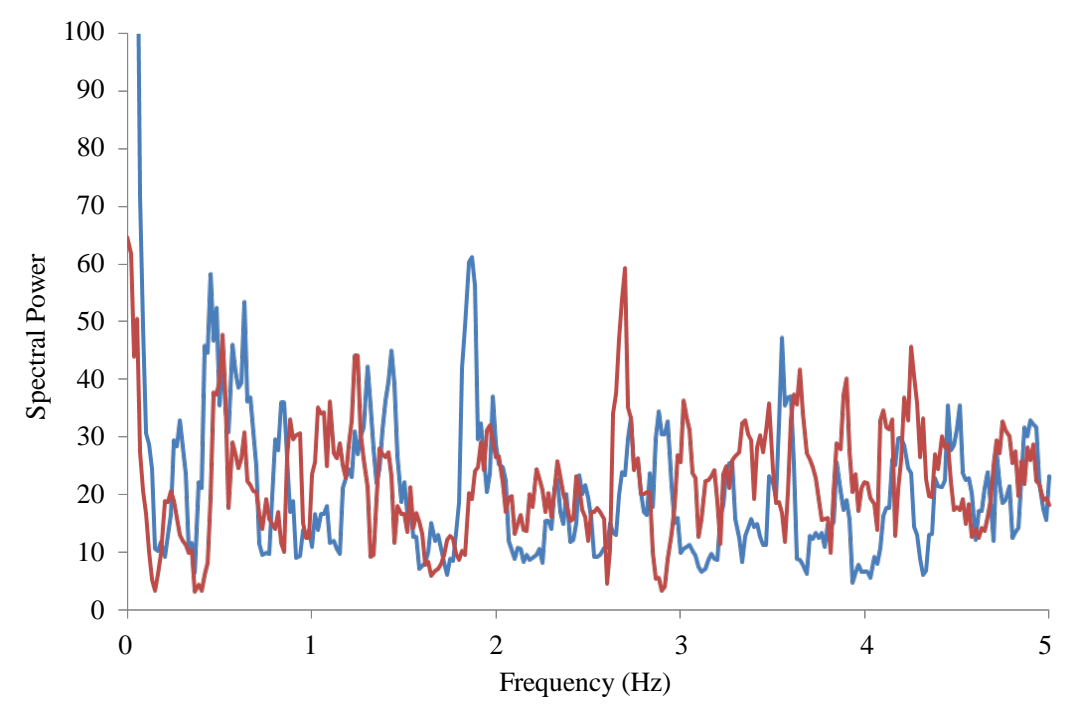

Figure 2. Spectral power denisties for the amplitude of photon emissions during baseline (rest) conditions for the left (blue) and right (red) hemispheres. Note the singular peak in power for frequency in the left hemisphere around $1.8 \mathrm{~Hz}$ (blue) and in the right hemisphere around $2.8 \mathrm{~Hz}$ (red).

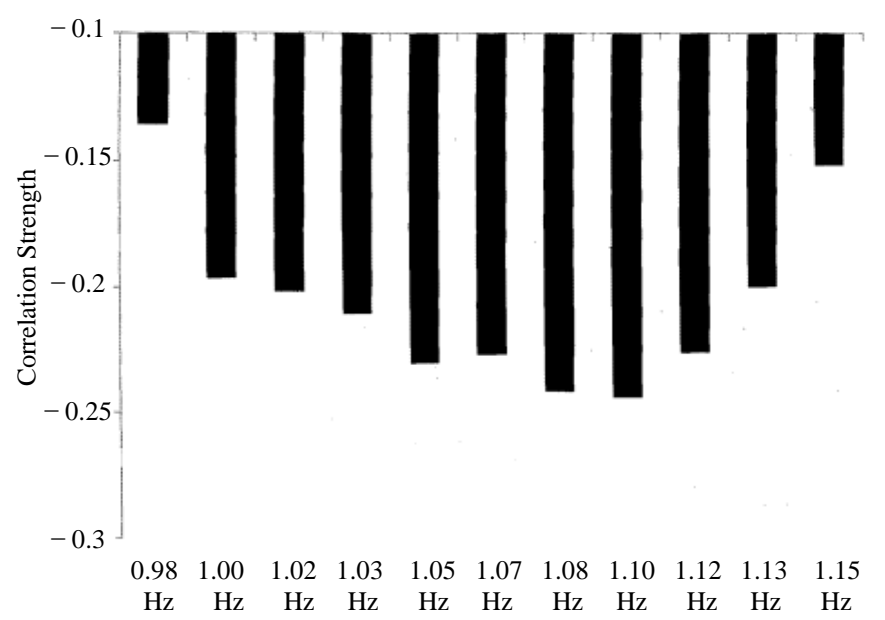

Figure 3. Strength of correlations between spectral power densities for different frequency lags. All lags between 0.98 and $1.15 \mathrm{~Hz}$ were statistically significant at the $\mathrm{p}<0.05$ level. 
would be have been about 800 to $890 \mathrm{~ms}$ which is within the range of the lag measured from the standardized scores of the power density of the photon emission every $100 \mathrm{~ms}$.

\section{Discussion}

These results support measurements from previous studies that photon emissions can be measured at distances more than direct contact to the human skull during a brief $(1 \mathrm{~min})$ baseline sampling if the person is sitting in a hyper-dark setting. There is strong evidence that the power densities of the photon emissions for each hemisphere are not temporally coherent but lagged by approximately $1 \mathrm{~s}$. This was evident from the lag analyses for the standardized photon counts as well as the results of the comparisons of the spectral densities for the left and right hemisphere of the volunteers.

The discrepancy could be considered a phase lag analogous to the Aharanov-Bohm phenomenon that has been attributed to electrons moving near a special magnetic field condition. The degree of phase shift is related to the intrinsic voltages and the time within the field. The relationship can be set to solve for $t$ (the time within the field) as:

$$
t=(\Psi \cdot \hbar) \cdot(V \cdot q)^{-1}
$$

where $\Psi$ is the phase lag; $\hbar$ is the modified Planck's constant; $V$ is the voltage and $q$ is the unit charge. If the phase lag is reflected by the ratio of $1.8 \mathrm{~Hz}$ (for the left hemisphere) and $2.8 \mathrm{~Hz}$ (for the right hemisphere), that is 0.64 , and the energy $V q$ is that associated with the median energy of the photons discerned by the photomultiplier units, $5 \times 10^{-19} \mathrm{~J}$, then $t$, the time each electron that might be related to the photon emission within the field is $1.34 \times 10^{-16} \mathrm{~s}$.

This duration is remarkably proximal to the time required for an electron to complete one Bohr orbit $(1.52 \times$ $10^{-16} \mathrm{~s}$ ). This is the same order of magnitude and comparable coefficient for the time required for a photon moving $1 / 3^{\text {rd }}$ the velocity of light in a vacuum to traverse a plasma cell membrane of the neuron which averages around $10 \mathrm{~nm}$. This convergence at least sets the conditions for the photon to intercalate with the completion of a single electron orbit. The energy associated with the mass of an electron moving at the square of the fine structure velocity in the time of one orbit is effectively Planck's regular constant, particularly when the ratio of the spin and orbital magnetic moment of the electron is accommodated.

In previous studies we had shown that the amplitude of the photon emissions from the right hemisphere but not the left hemisphere increased during imagining white light but not during engagement of more mundane cognitive tasks [1]. For these no-imagine, imagine, no-imagine, and imagine procedures, the net increase compared to the previous no imagine interval was about $5 \times 10^{-12} \mathrm{~W} \cdot \mathrm{m}^{-2}$. We had estimated that based upon the cross sectional area of the lateral cerebral hemispheres (about $10^{-2} \mathrm{~m}^{2}$ ), this would be equivalent to about $10^{-14} \mathrm{~J}$ per second. Assuming the neurons contributing to this energy were each discharging on average at $10 \mathrm{~Hz}$ and each action potential involved $10^{-20} \mathrm{~J}$, the source population would be in the order of $10^{6}$ to $10^{7}$ neurons.

Aboitiz et al. [13] as confirmed by Bojak and Liley [14] indicated that the numbers of unmyelinated axons within the human corpus callosum between 0.4 and $1 \mu \mathrm{m}$ diameter would involve about $1.6 \times 10^{7}$ neurons compared to the estimated 200 million within the entire structure. This would involve a maximum of about $6.5 \%$ of the total population of fibers. For comparison these diameters if they were electromagnetic wavelengths (400 to $1000 \mathrm{~nm}$ ) would include the range of visible photons. Dotta et al. [15] [16], while measuring malignant cells that had been removed from incubation, showed that the typical wavelengths associated with metabolic and replication activity concentrated within the interfaces between ultraviolet-visible and visible-infrared.

We suggest that the source of the photon emission interaction between the left and right hemispheres may primarily involve the non-myelinated axons with widths between 400 and $800 \mathrm{~nm}$. If the approximately $6.5 \%$ of the total corpus callosal fibers were mediating the effect and were determining effect size then any correlation between the two hemispheres would be the square root of that value or equivalent to a $r$ value of 0.25 . This is within error variation of what we measured in this experiment.

There are relatively sound biophysical bases to this number. Assuming the coherence of voltage across the major area of the cerebral cortices is in the order of $0.2 \mathrm{mV} \cdot \mathrm{m}^{-1}$ or $2 \times 10^{-4} \mathrm{~V} \cdot \mathrm{m}^{-1}[17]$ and the extracellular fluid resistance of $2 \Omega \cdot \mathrm{m}$ [18] the current density would be $10^{-4} \mathrm{~A} \cdot \mathrm{m}^{-2}$. Over the total surface area of the cerebral cortices of $1.8 \times 10^{-1} \mathrm{~m}^{2}$ the electric current would be $1.8 \times 10^{-5} \mathrm{~A}$. Given that there is $1.6 \times 10^{-19} \mathrm{~A} \cdot \mathrm{s}$ per unit charge, this means that a total of $1.1 \times 10^{14}$ charges per second would be involved. Typical estimates average $10^{6}$ 
to $10^{7}$ charges per action potential. Hence $10^{14}$ charges per s would be equivalent to $10^{6}$ to $10^{7}$ axons discharging between 1 to $10 \mathrm{~Hz}$. Assuming the dominant contribution from $\sim 4 \times 10^{7}$ action potentials per second each with a unit energy of $10^{-20} \mathrm{~J}$ the energy [19] would be $10^{-13} \mathrm{~J} \cdot \mathrm{s}^{-1}(\mathrm{~W})$. When distributed over the area of the average cerebral cortices $\left(1.8 \times 10^{-1} \mathrm{~m}^{2}\right)$ the radiant flux density would be $\sim 2 \times 10^{-12} \mathrm{~W} \cdot \mathrm{m}^{-2}$ which would be within the range of the measurements recorded in this study.

The repeated observations in previous experiments [1] [3] that instructions to imagine or not imagine types of imagery was associated with increased photon emissions from the right side of the brain and not the left side of the brain [1] minimized the likelihood of artifact. In addition, we had found a powerful correlation accommodating more than $80 \%$ of the variance between quantitative electroencephalographic power within the beta range over the left prefrontal lobes of the participants and the power density of the photon emissions from the right hemisphere during the experiment [1]. One interpretation of this strong association is that intention or some factor associated with this process might influence the power density of biophoton emissions from the right hemisphere.

In the present experiment the lag of the peak correlation strength between the power density of photon emissions from the left and right hemisphere during baseline conditions when both measures occur simultaneously suggests that either each hemisphere is operating independently or that a discrete amount of time is required before the processes that are responsible for photon emissions in one hemisphere affect the processes associated with photon emissions from the other. Whatever process is involved the strongest cross-spectral correlation of about $r=0.25$ indicates that only $6.5 \%$ of the variance in shared distributions of power density occurs between the two hemispheres when the optimal lag is implemented.

We employed the ratio of $1.8 \mathrm{~Hz}$ and $2.8 \mathrm{~Hz}$ spectral power density peaks for the left and right hemispheres, respectively, to obtain the inferred ratio for the phase shift in an Aharanov-Bohm effect if it were valid at macroscopic contexts [20]. The equivalent time would sufficiently overlap with the latency for a photon to traverse a neuronal plasma membrane and the orbit of a single electron. This creates the condition for an exchange of quantum bits of information moving from the membrane into space or from space into the membrane. We consider the upper boundary ( $433 \mathrm{~ms}$ ) of the latency required for transmission along unmyelinated axons between the two hemispheres to be supportive of our absolute lag in hemispheric time $(900 \mathrm{~ms})$ if a complete circuit is considered. Considering the average transmission velocity to be $8.7 \mathrm{~mm}$ per $\mathrm{ms}$ per $\mu \mathrm{m}$ and the median width of an axon to be $500 \mathrm{~nm}$, the single traversing time would be 25 to $30 \mathrm{~ms}$ or 33 to $40 \mathrm{~Hz}$. This is the classic frequency band associated with human consciousness and awareness.

\section{Acknowledgements}

Thanks to Dr. W. E. Bosarge, Jr., Chairman, Quantlab LLC for financial support. The technical skills of Viger M. Persinger are appreciated.

\section{References}

[1] Dotta, B.T., Saroka, K.S. and Persinger, M.A. (2012) Increased Photon Emission from the Head While Imagining Light in the Dark Is Correlated with Changes in Electroencephalographic Power: Support for Bókkon's Biophoton Hypothesis. Neuroscience Letters, 513, 151-154. http://dx.doi.org/10.1016/j.neulet.2012.02.021

[2] Dotta, B.T., Buckner, C.A., Lafrenie, R.M. and Persinger, M.A. (2011) Photon Emissions from Human Brain and Cell Culture Exposed to Distally Rotating Magnetic Fields Shared by Separate Light-Stimulated Brains and Cells. Brain Research, 1388, 77-88. http://dx.doi.org/10.1016/j.brainres.2011.03.001

[3] Saroka, K.S., Dotta, B.T. and Persinger, M.A. (2013) Concurrent Photon Emission, Changes in Quantitative Brain Activity over the Right Hemisphere, and Alterations in the Proximal Geomagnetic Field While Imagining White Light. International Journal of Life Sciences and Medical Research, 3, 30-34. http://dx.doi.org/10.5963/LSMR0301005

[4] Tilbury, R.N. and Quickenden, T.I. (1988) Spectral and Time Dependence Studies of the Ultraweak Bioluminescence Emitted by the Bacterium Escherichia coli. Photochemistry and Photobiology, 47, 145-150. http://dx.doi.org/10.1111/j.1751-1097.1988.tb02704.x

[5] Inaba, J. (1988) Super-High Sensitivity Systems for Detection and Spectral Analysis of Ultraweak Photon Emission from Biological Cells and Tissues. Experentia, 44, 530-559. http://dx.doi.org/10.1007/BF01953302

[6] Isojima, Y., Isoshima, T., Nagai, K., Kikuchi, K. and Nakagawa, H. (1995) Ultraweak Biochemiluminescence Detected from Rat Hippocampal Slices. NeuroReprot, 6, 658-660. http://dx.doi.org/10.1097/00001756-199503000-00018 
[7] Sun, Y., Wang, C. and Dai, J. (2010) Biophotons as Neural Communication Signals Demonstrated by in Situ Biophoton Autography. Photochemical and Photobiological Sciences, 9, 315-322. http://dx.doi.org/10.1039/b9pp00125e

[8] Popp, F.-A. (1988) Biophoton Emission. Experientia, 44, 543-544. http://dx.doi.org/10.1007/BF01953300

[9] Popp, F.-A. (1979) Photon Storage in Biological Systems. Electromagnetic Bioinformation. Urban and Schwarzenberg, N.Y., 123-149.

[10] Van Wijk, R. and Schamhart, D.H.J. (1988) Regulatory Aspects of Low Intensity Photon Emission. Experientia, 44, 586-593. http://dx.doi.org/10.1007/BF01953306

[11] Van Wijk, R., Van Wijk, E.P. and Bajpai, R.P. (2006) Photocount Distributions of Photons Emitted from Three Sites of the Body. Journal of Photochemistry and Photobiology B: Biology, 84, 46-55. http://dx.doi.org/10.1016/j.jphotobiol.2006.01.010

[12] Saroka, K.S., Caswell, J.M., Lapointe, A. and Persinger, M.A. (2014) Greater Electroencephalographic Coherence between Left and Right Temporal Lobe Structures during Increased Geomagnetic Activity. Neuroscience Letters, 560, 126-130. http://dx.doi.org/10.1016/j.neulet.2013.11.024

[13] Aboitiz, F., Scheibel, A.B., Fischer, R.S. and Zaidel, E. (1992) Fibre Composition of the Corpus Callosum. Brain Research, 598, 143-115. http://dx.doi.org/10.1016/0006-8993(92)90178-C

[14] Bojak, I. and Liley, D.T.J. (2010) Axonal Velocity Distributions in Neural Field Equations. PLoS Computational Biology, 6, e1000653. http://dx.doi.org/10.1371/journal.pcbi.1000653

[15] Dotta, B.T., Buckner, C.A., Cameron, D., Lafrenie, R. and Persinger, M.A. (2011) Photon Emissions from Cell Cultures: Biochemical Evidence for the Plasma Membrane as the Primary Source. General Physiology and Biophysics, 30, 301-309.

[16] Dotta, B.T., Murugan, N.J., Karbowski, L.M., Lafrenie, R.M. and Persinger, M.A. (2014) Shifting Wavelengths of Ultraweak Photon Emissions from Dying Melanoma Cells: Their Chemical Enhancement and Blocking Are Predicted by Cosic's Theory of Resonant Recognition Model for Macromolecules. Naturwissenschaften, 101, 87-94. http://dx.doi.org/10.1007/s00114-013-1133-3

[17] Saroka, K.S. and Persinger, M.A. (2014) Quantitative Evidence for Direct Effects between Earth-Ionosphere Schumann Resonances and Human Cerebral Cortical Activity. International Letters of Chemistry, Physics and Astronom, 20, 166-194. http://dx.doi.org/10.18052/www.scipress.com/ILCPA.39.166

[18] Barnes, F.S. (1986) Interaction of DC Electric Fields and Living Matter. In: Polk, C. and Postow, E., Eds., Handbook of Biological Effects of Electromagnetic Fields, CRC Press, Boca Raton, 99-120.

[19] Persinger, M.A. (2010) 10 - 20 Joules as a Neuromolecular Quantum in Medicinal Chemistry: An Alternative Approach to Myriad Molecular Pathways? Current Medicinal Chemistry, 17, 3094-3098. http://dx.doi.org/10.2174/092986710791959701

[20] Persinger, M.A. and Koren, S.A. (2016) The Aharanov-Bohm Phase Shift and Magnetic Vector A Potential Could Accommodate the Optical Coupler, Digital-to-Analogue Magnetic Field Excess Correlations of Photon Emissions within Living Aqueous Systems. Journal of Advances in Physics, 11, 3333-3339. 